\title{
In Vitro and In Vivo Characterization of a Murine Cytomegalovirus with a Mutation at Open Reading Frame m166
}

\author{
Jiaming Zhu, Jennifer Chen, Rong Hai, Tuong Tong, Jianqiao Xiao, Xiaoyan Zhan, \\ Sangwei Lu, and Fenyong Liu* \\ Division of Infectious Diseases, School of Public Health, University of California, Berkeley, California 94720
}

Received 11 September 2002/Accepted 22 November 2002

\begin{abstract}
We have recently generated a pool of murine cytomegalovirus (MCMV) mutants by using a Tn3-based transposon mutagenesis approach. In this study, one of the mutants, Rvm166, which contained the transposon sequence at open reading frame $\mathrm{m} 166$, was characterized both in tissue culture and in immunocompetent BALB/c mice and immunodeficient SCID mice. The viral mutant replicated as well as the wild-type Smith strain in vitro in NIH 3 T3 cells, whereas the transposon insertion precluded the expression of $>65 \%$ of the m166 open reading frame. Compared to the wild-type strain and a rescued virus that restored the m166 region, the viral mutant was significantly attenuated in growth in both BALB/c and SCID mice that were intraperitoneally infected with the viruses. At 21 days postinfection, the titers of the viral mutant in the salivary glands, lungs, spleens, livers, and kidneys of the infected SCID mice were lower than the titers of the Smith strain and the rescued virus by about 30,000-, 10,000-, 1,000-, 300-, and 800-fold, respectively. Moreover, the virulence of the mutant virus appears to be severely attenuated because no death was found in SCID mice infected with the viral mutant up to 90 days postinfection, whereas all of the animals infected with the wild-type and rescued viruses died at 27 days postinfection. Our results suggest that m166 probably encodes a virulence factor and is required for MCMV virulence in killing SCID mice and for optimal viral growth in vivo.
\end{abstract}

Human cytomegalovirus (HCMV), a human betaherpesvirus, is one of the most common opportunistic pathogens associated with immunoimmature or immunocompromised individuals $(26,30)$. It is the leading viral cause of birth defects, affecting about 7,000 infants yearly in the United States alone (12). Moreover, HCMV infection continues to be a major cause of morbidity and mortality in bone marrow and solid organ transplant recipients and is among the most common opportunistic complications found in AIDS patients $(26,30)$. More recently, HCMV infection has been proposed to be associated with transplant vascular sclerosis, as well as atherosclerosis $(3,9,24,42,50)$. Understanding the mechanism of HCMV pathogenesis and the function of viral genes in HCMV-related diseases is necessary for developing new drugs and novel strategies for the treatment and prevention of HCMV infections.

HCMV has a genome of about $230 \mathrm{~kb}$ and a coding capacity of more than 200 open reading frames $(6,26)$. This virus only propagates in human or chimpanzee cells but not in cells derived from other species. Species restriction and lack of readily available seronegative primates has prevented the study of HCMV in any animal model. Murine cytomegalovirus (MCMV) provides an excellent model for studying the biology of cytomegalovirus (CMV) infection (16, 18, 26, 30). MCMV infection of mice resembles its human counterpart in many ways with respect to pathogenesis during acute infection, establishment of latency, and reactivation after immunosuppression or transplantation $(16,18,26,30)$. The MCMV genome is

\footnotetext{
* Corresponding author. Mailing address: Division of Infectious Diseases, School of Public Health, 140 Warren Hall, University of California, Berkeley, CA 94720. Phone: (510) 643-2436. Fax: (510) 643-9955. E-mail: liu_fy@uclink4.berkeley.edu.
}

also $230 \mathrm{~kb}$ long and is predicted to encode more than 170 open reading frames, 78 of which have extensive homology to those of $\operatorname{HCMV}(6,32)$. A complete understanding of the biology of MCMV and the function of its genes may provide insight into the pathogenesis of HCMV.

One of the most powerful approaches for studying the function of virus-encoded genes is to introduce mutations into the viral genome and to screen viral mutants in both tissue culture and animals for possible growth defects in vitro and in vivo. The construction of mutants of CMV and related herpes simplex virus 1 (HSV-1) has been reported by using site-directed homologous recombination and transposon-mediated insertional mutagenesis $(17,27,40,44,46)$. More recently, the MCMV genome, as well as the genomes of other herpesviruses, have been cloned into a bacterial artificial chromosome (BAC), and viral mutants were successfully generated from the BAC-based viral genome by a bacterial mutagenesis procedure $(2,4,10,25,36,38,39,41,45)$. These studies have greatly facilitated the identification of the functions of viral genes in tissue culture and in animals.

Many of the CMV genes have been found to be dispensable for growth in cultured cells. Their presence in the viral genome indicates that they are probably needed to perform functions only involved in modulating the interactions between the virus and its respective human or animal hosts. Some of these genes from a particular CMV (e.g., MCMV) might have evolved with its respective host and interact with specific components of the host and, therefore, are unique and may not share significant sequence homologies with CMVs from other species (e.g., HCMV). Studies of the function of these species-specific viral genes will provide insight into the general mechanism of how CMV interacts with its respective host and establishes its infection. For example, HCMV US11, a nonessential protein, 
functions to downregulate the expression and presentation of the major histocompatibility complex class I molecules (47). Meanwhile, MCMV open reading frame m131, which is dispensable for viral replication, encodes a chemokine that promotes monocyte-associated viremia for viral dissemination in vivo $(11,22,35)$. Thus, studies of viral mutants carrying mutations in genes that are found to be dispensable in tissue culture, including the species-specific open reading frames, are valuable to the understanding of gene function in viral pathogenesis and virus-host interactions, including tissue tropism and virulence.

We have previously generated a pool of MCMV mutants by using a Tn3 transposon-mediated shuttle mutagenesis system $(48,49)$. In this approach, the transposon is randomly inserted into the MCMV genomic DNA fragments in a plasmid library in Escherichia coli. Regions bearing an insertion mutation are then transferred to the MCMV genome by cotransfecting the plasmid library and purified MCMV genomic DNA into NIH 3 T3 cells. Characterization of one of the viral mutants (e.g., Rvm09) in NIH 3 T3 cells and in both BALB/c and SCID mice indicated that the presence of the transposon sequence per se in the viral genome does not significantly affect viral replication and growth in vitro and in vivo (49). In the present study, we characterized an MCMV mutant, Rvm166, which contains a transposon insertion in open reading frame m166. The function of m166 in viral replication and pathogenesis is currently unknown. Indeed, this open reading frame has not been extensively characterized transcriptionally or translationally. The $\mathrm{m} 166$ sequence is uniquely found in MCMV, and there is no sequence homologue of m166 in HCMV, suggesting that m166 is probably involved in species-specific interactions between MCMV and its natural host, mice. Our results provide the first direct evidence to suggest that a disruption of the m166 open reading frame leads to severe attenuation of viral virulence and deficient growth in vivo. When the mutant virus was used to infect immunocompetent $\mathrm{BALB} / \mathrm{c}$ mice and immunodeficient SCID mice intraperitoneally, the viral titers in the salivary glands, lungs, spleens, livers, and kidneys were significantly lower than those in mice inoculated with the wild-type virus and with a revertant virus in which the mutation was rescued and the $\mathrm{m} 166$ open reading frame was restored. Moreover, the viral mutant was severely attenuated in killing SCID mice. All of the SCID mice infected with Rvm166 remained alive for at least 90 days postinfection, whereas those infected with the wild-type and rescued virus died within 27 days postinfection. These results suggest that $\mathrm{m} 166$ is a viral determinant for MCMV pathogenecity and is required for viral virulence and growth in vivo.

\section{MATERIALS AND METHODS}

Animals. The immunocompetent BALB/c-ByJ mice and immunodeficient CB17 SCID mice were purchased from the Jackson Laboratory (Bay Harbor, Maine) and National Cancer Institute (Frederick, Md.), respectively, and used at 3 to 5 weeks of age. The animals were housed in microisolator cages and fed and watered ad libitum throughout the experiments. Mice were acclimatized for 2 to 3 days prior to infection.

Viral virulence in SCID mice. The virulence of the viruses was studied by determining the mortality rates of animals infected with the Smith strain, Rvm166, or Rqm166. Male CB17 SCID mice (4 to 6 weeks old; five animals per group) were infected intraperitoneally with $10^{4} \mathrm{PFU}$ of each virus. The viral inoculum used for the infection of the animals was prepared by growing the viruses in NIH $3 \mathrm{~T} 3$ cells. The animals were observed twice daily, and the mortality of the infected animals was monitored for at least 90 days postinfection, and the survival rates for each virus were determined.

Viruses and cells. The MCMV Smith strain, mouse NIH 3 T3 cells, and STO cells were obtained from the American Type Culture Collection (ATCC; Rockville, Md.). The Smith strain, viral mutant Rvm166, and rescued virus Rqm166 were grown in NIH 3 T3 cells (ATCC CRL-1658) in Dulbecco modified Eagle medium (DMEM) supplemented with $10 \%$ Nu-Serum (Collaborative Research, Inc., Waltham, Mass.), $100 \mathrm{U}$ of penicillin/ml, $100 \mu \mathrm{g}$ of streptomycin/ml, and $0.1 \mathrm{mM}$ minimal essential medium amino acids (Gibco-BRL, Grand Island, N.Y.). STO cells (ATCC CRL-1503) were grown in DMEM supplemented with $10 \%$ fetal bovine serum (Gibco-BRL), $100 \mathrm{U}$ of penicillin/ml, and $100 \mu \mathrm{g}$ of streptomycin $/ \mathrm{ml}$. Cell cultures were maintained at $37^{\circ} \mathrm{C}$ in a humidified incubator with $5 \% \mathrm{CO}_{2}$

Construction of transposon-containing MCMV mutants and generation of the rescued virus. The transposon (Tn3gpt), which is derived from the Tn 3 transposon from $E$. coli $(5,34,37)$, contains the expression cassette encoding guanine phosphoribosyltransferase ( $g p t$ ) (which contains the gpt coding sequence driven by a promoter and a transcription termination signal) and an additional transcription termination site (Fig. 1A) (48). Construction of an MCMV genomic subclone pool and the procedure of transposon-based shuttle mutagenesis to generate a pool of MCMV DNA fragments (MCMV-Tn3gpt) containing a Tn3gpt insertion were performed as described by Zhan et al. (48). To generate a pool of MCMV mutants that contained the transposon sequence, full-length MCMV genomic DNA and plasmid DNA containing MCMV-Tn3gpt fragments were cotransfected into NIH 3 T3 cells by using a calcium phosphate precipitation protocol (Gibco-BRL). The recombinant MCMV was selected in the presence of mycophenolic acid $(25 \mu \mathrm{g} / \mathrm{ml}$; Gibco-BRL) and xanthine $(50 \mu \mathrm{g} / \mathrm{ml}$; Sigma, St. Louis, Mo.) and plaque purified three times according to the protocol described previously (49). In order to determine the location of the transposon insertion within the viral genome and to identify the genes that were disrupted by the transposon insertion, viral DNA was purified and directly sequenced by using the primer FL110PRIM (5'-GCAGGATCCTATCCATATGAC-3') by the fmol cycle sequencing kit (Promega, Inc., Madison, Wis.).

To construct the rescued virus Rqm166, the full-length genomic DNA of Rvm166 was isolated from virus-infected cells as described previously (49). The full-length intact Rvm166 genomic DNA and the DNA sequence that contained the coding sequence of m166 were subsequently cotransfected into mouse cells by using a calcium phosphate precipitation protocol (Gibco-BRL). The recombinant virus was selected in STO cells in the presence of $25 \mu \mathrm{g} / \mathrm{ml}$ of 6-thioguanine (Sigma) and purified by six rounds of amplification and plaque purification, according to the protocol described previously (14). For each cotransfection, several viral plaques were picked and expanded. Viral stocks were prepared by growing the viruses in roller bottles of NIH $3 \mathrm{~T} 3$ cells.

Growth of the viruses in vitro. The growth kinetics of the viruses was determined as described previously (49). Briefly, NIH 3 T3 cells grown to 60 to $75 \%$ confluence were inoculated at a multiplicity of infection (MOI) of 0.5 or 5 of the virus. At $0,1,2,4$, and 7 days postinfection, the infected cells, together with medium were harvested and an equal volume of $10 \%$ skim milk, was added before sonication. The virus titer was determined by plaque assays in NIH 3T3 cells. The values of the viral titers were the average of triplicate experiments.

Northern analyses of recombinant viruses. Cells were infected with virus at an MOI of 5 and harvested at different time points postinfection. In the experiments to assay the expression of immediate-early transcripts, cells were pretreated with $100 \mu \mathrm{g}$ of cycloheximide (Sigma)/ml and then infected with viruses and harvested at $6 \mathrm{~h}$ postinfection (21). Total cytoplasmic RNA was isolated from NIH 3T3 cells infected with the viruses as described previously (20). Viral RNAs were separated in 0.8 to $1 \%$ agarose gels that contained formaldehyde, transferred to a nitrocellulose membrane, hybridized with ${ }^{32} \mathrm{P}$-radiolabeled DNA probes that contained the MCMV sequences, and finally analyzed with a STORM840 phosphorimager. The DNA probes used for Northern analyses were generated by PCR by using viral DNA as the template and radiolabeled with a random primer synthesis kit in the presence of $\left[\alpha^{3}{ }^{32} \mathrm{P}\right] \mathrm{dCTP}$ (Boehringer Mannheim, Indianapolis, Ind.). The 5' PCR primers used in the construction of DNA probes for the Northern analysis of the m166 and M25 transcripts were JMm166down1 (5'-G TGAACGCCTACACGGG-3') and M25-5'NDS (5'-CGACGACGATGACGA CGATG-3'), respectively. The $3^{\prime}$ PCR primers used were JMm166down2 (5'-G TTCACGAGTATCTCATAGGC-3') and M25-3'NDS (5'-GTCCTGACCGCT CACTACAC-3'), respectively.

Southern analysis of recombinant viruses. Viral DNA was purified from NIH 3 T3 cells infected with the viruses as described previously $(44,48)$. Briefly, cells that exhibited $100 \%$ cytopathic effect were washed with phosphate-buffered saline and then lysed with a solution that contained sodium dodecyl sulfate and 


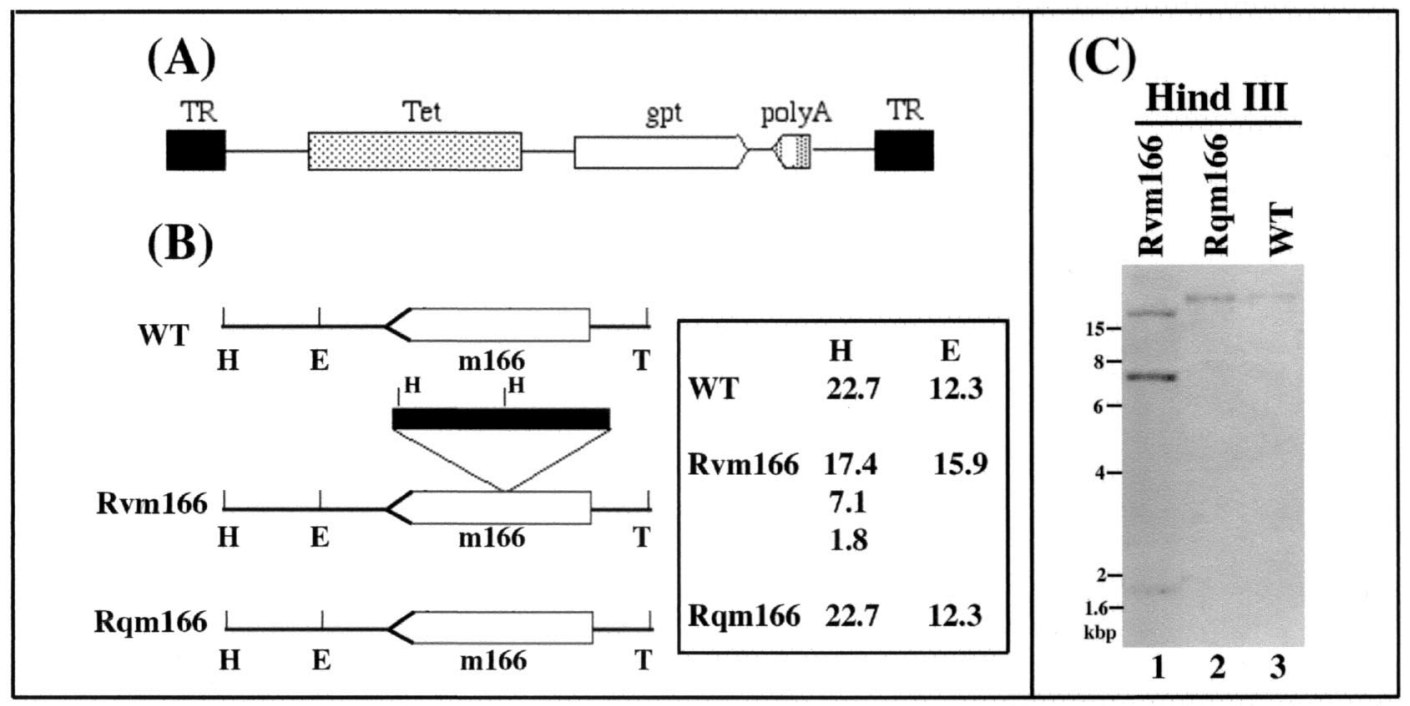

FIG. 1. (A) Structure of the transposon construct used for mutagenesis. TR, terminal repeat; Tet, tetracycline resistance gene; gpt, gene that encodes guanine phosphoribosyltransferase (gpt); poly(A), transcription termination signal; $\mathrm{T}$, the $3^{\prime}$ terminus of viral genome. (B) Transposon insertion in the recombinant virus. The m166 coding sequence is shown as an open arrow, whereas the transposon is represented as a filled bar. The numbers represent the sizes of the DNA fragments of the viruses that were generated by digestion with HindIII (H) and EcoRI (E). (C) Southern analyses of the recombinant viruses. The DNA fractions were isolated from cells infected with the wild-type (WT) virus, Rvm166 (Rvm166) or Rqm166 (Rqm166). The DNA samples $(20 \mu \mathrm{g})$ were digested with HindIII (H), separated on $0.8 \%$ agarose gels, transferred to a Zeta-Probe membrane, and hybridized to a DNA probe that contained the MCMV DNA fragment inserted with the transposon sequence.

proteinase K. The genomic DNA was purified by extraction with phenol-chloroform, followed by precipitation with 2-propanol. The DNA was then digested with HindIII or EcoRI, separated on agarose gels (0.8 to $1 \%)$, transferred to Zeta-Probe nylon membranes (Bio-Rad, Hercules, Calif.), hybridized with the ${ }^{32} \mathrm{P}$-radiolabeled DNA probes that contained the transposon and the MCMV m166 sequence, and finally analyzed with a STORM840 phosphorimager (49). The labeled DNA probes were prepared by random primer synthesis (Boehringer Mannheim, Indianapolis, Ind.).

Studies of the growth of the viruses in animals. The viral inoculum used for the infection of animals was prepared by growing the viruses in NIH 3 T3 cells. Three- to four-week-old male BALB/c-ByJ mice or four- to six-week-old CB17 SCID mice were infected intraperitoneally with $10^{4} \mathrm{PFU}$ of each virus. The infected animals were sacrificed at $1,3,7,10,14$, and 21 days postinoculation. The salivary glands, lungs, spleens, livers, and kidneys were harvested and sonicated as a $10 \%(\mathrm{wt} / \mathrm{vol})$ suspension in a 1:1 mixture of DMEM medium and $10 \%$ skim milk. The sonicates were stored at $-80^{\circ} \mathrm{C}$ until plaque assays were performed. For each time point, at least three animals were used as a group and infected with the same virus.

Plaque assays were performed in NIH 3T3 cells plated overnight to about 60 to $75 \%$ confluence in six-well cluster plates (Costar, Corning, N.Y.). Tenfold serial dilutions of virus in $1 \mathrm{ml}$ of DMEM were inoculated onto each well of NIH $3 \mathrm{~T} 3$ cells. After $120 \mathrm{~min}$ of incubation at $37^{\circ} \mathrm{C}$ in a $5 \% \mathrm{CO}_{2}$ incubator, the cells were washed with DMEM, and then overlaid with DMEM containing $1 \%$ lowmelting-point agarose (Sigma). Viral plaques were counted after 4 to 6 days under an inverted microscope. The titer of each sample was determined in triplicate, and the titer of the sample was expressed as the average of the three values. Viral titers were recorded as the PFU/milliliter of organ homogenate. The limit of virus detection in the organ homogenates was $10 \mathrm{PFU} / \mathrm{ml}$ of the sonicated mixture. Samples that were negative at a $10^{-1}$ dilution were designated as having a titer value of $10\left(10^{1}\right) \mathrm{PFU} / \mathrm{ml}$.

\section{RESULTS}

Isolation of a MCMV mutant containing the transposon insertion at open reading frame $\mathbf{m} 166$. We have used an Escherichia coli Tn3-based transposon mutagenesis system to construct a pool of MCMV mutants (48). Figure 1A shows the structure of the transposon used to generate MCMV mutants.
The transposon contains the expression cassette coding for guanine phosphoribosyltransferase $(g p t)$, which includes the gpt coding sequence driven by a simian virus 40 promoter and a transcription termination signal, and an additional transcription termination site (48). The gpt expression cassette and the additional poly(A) signal in the transposon are in opposite orientations (Fig. 1A). Therefore, the expression of nearby genes that may share a common poly(A) signal with the target gene is not disrupted. To generate a pool of MCMV mutants, a library of MCMV genomic fragments containing randomly inserted Tn3gpt sequence were first constructed as described previously and then cotransfected with the full-length genomic DNA of the wild-type virus (Smith strain) into mouse NIH 3T3 cells where homologous recombination took place. The cells that harbored the progeny viruses expressing the gpt gene were selected for growth in the presence of mycophenolic acid and xanthine $(14,28,44)$. After multiple rounds of selection and plaque purification, individual recombinant viruses were isolated and the location of the inserted transposon was determined by directly sequencing the genomic DNA of the recombinants. One of the recombinant viruses contained the transposon sequence inserted within open reading frame m166 and is designated as Rvm166 (Fig. 1B). Sequencing analyses of the junction between the transposon and the viral sequence revealed that the location of the transposon in Rvm166 is at nucleotide position 225172 (amino acid residue 130 of the 382 -amino-acid open reading frame) in reference to the genome sequence of the wild-type Smith strain (32) (Fig. 1B and data not shown).

Characterization of $\mathrm{Rvm} 166$ and rescued virus $\mathrm{Rqm166}$ by Southern analysis. The genomic structure of Rvm166 and the location of the transposon insertion in the viral genome were 
examined by Southern blot hybridization with a DNA probe containing both the transposon and the viral sequence (Fig. 1B and C). When the viral DNA samples were digested with HindIII and subjected to Southern analyses, a small fragment of $1.8 \mathrm{~kb}$ representing the gpt gene was detected, indicating the presence of the transposon sequence within the viral genome (Fig. 1C, lane 1). This finding was further supported by the results of Southern analyses of the Rvm166 DNA samples digested with another restriction enzyme, EcoRI. In these experiments, the genomic fragments containing the transposon were found to be larger than those of the wild-type virus by 3.6 $\mathrm{kb}$, which is the size of the transposon (data not shown; see Fig. 7).

The Southern blots also showed that the stocks of the mutant virus were pure and free of the wild-type strain since the hybridizing DNA fragments from the mutant did not comigrate with those of the wild-type Smith strain (Fig. 1C, lanes 1 and 3; see Fig. 7, lanes 1 and 2). For example, the hybridization patterns of the Rvm166 and Smith strain DNAs digested with HindIII gave rise to three $(17.4,7.1$, and $1.8 \mathrm{~kb})$ and one DNA bands (22.7 kb), respectively (Fig. 1C, lanes 1 and 3). Meanwhile, the hybridized species $(15.9 \mathrm{~kb})$ of the EcoRI-digested Rvm166 DNA migrated differently from that $(12.3 \mathrm{~kb})$ of the wild-type viral DNA digested with the same enzyme (Fig. 1B; see also Fig. 7, lanes 1 and 2). The sizes of the hybridized DNA fragments (Fig. 1C and 7) were consistent with the predicted digestion patterns of the recombinant virus based on the MCMV genomic sequence (32) and the location of the transposon insertion in the viral genome as determined by sequence analysis (Fig. 1B). The restriction enzyme digestion patterns of the regions of the mutant genomic DNA other than the transposon insertional site appeared to be identical to those of the parental Smith strain, as indicated by ethidium bromide staining of the digested DNAs (data not shown). This observation suggests that regions of the viral genome other than that containing the transposon insertion remained intact in this MCMV mutant.

It has been shown that spontaneous mutations within the viral genome, including deletions and rearrangements, can be generated during construction of viral mutants by using a homologous recombination approach (23; G. Abenes, X. Zhan, M. Lee, and F. Liu, unpublished results). Moreover, a spontaneous deletion of the $3^{\prime}$-terminal region of the viral genome has been reported (1). To exclude the possibility that the phenotype observed with Rvm166 might be due to some other adventitious mutations in the genome of the viral mutant rather than due to the disruption of the m166 open reading frame, a rescued virus, Rqm166, was derived from Rvm166, by restoration of the wild-type m166 sequence in Rvm166 (Fig. 1B). Construction of the rescued virus was carried out by using a procedure similar to that used for generating the viral mutant. A DNA fragment that contained the m166 coding region was cotransfected with the full-length Rvm166 genomic DNA into mouse cells and the recombinant viruses were selected in STO cells in the presence of 6-thioguanine, which selects against gpt expression $(14,28)$. The rescued virus, designated as Rqm166, which did not express the gpt protein and no longer contained the transposon, was isolated after multiple rounds of selection and plaque purification.

Southern hybridization analyses with DNA probes contain-

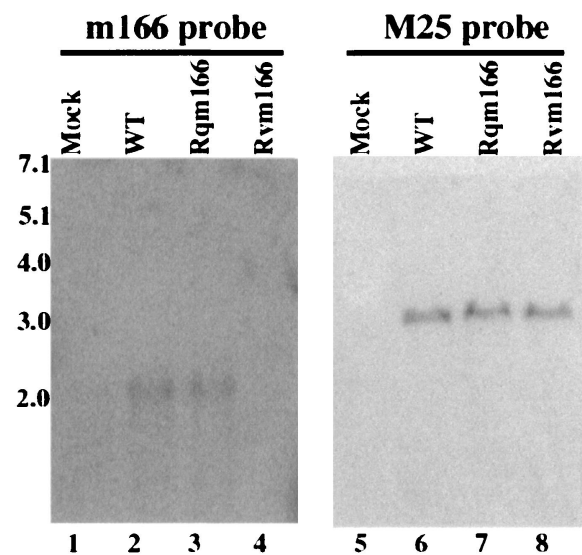

FIG. 2. Northern analyses of the RNA fractions isolated from cells that were mock infected (lanes 1 and 5) or infected with the wild-type virus (WT) (lanes 2 and 6), Rqm166 (lanes 3 and 7), and Rvm166 (lanes 4 and 8 ). A total of $5 \times 10^{6} \mathrm{NIH} 3 \mathrm{~T} 3$ cells were infected with each virus at an MOI of 5 PFU per cell, and cells were harvested at $24 \mathrm{~h}$ postinfection. RNA samples $(30 \mu \mathrm{g})$ were separated on agarose gels that contained formaldehyde, transferred to a nitrocellulose membrane, and hybridized to a ${ }^{32} \mathrm{P}$-radiolabeled probe that contained the sequence of m166 probe (lanes 1 to 4 ) or M25 probe (lanes 5 to 8 ).

ing the transposon and the viral sequence were used to characterize the genomic structure of Rqm166 and determine whether the m166 region was restored (Fig. 1B and C). Analysis of the Rqm166 DNA samples digested with HindIII and EcoRI showed that the sizes of the hybridized DNA fragments for Rqm166 were identical to those of the hybridized fragments for Smith strain and were different from those for Rvm166 (Fig. 1C, lane 2, and data not shown). These results indicated that Rqm166 did not contain the transposon sequence and that the m166 region was restored. Moreover, ethidium bromide staining of the digested DNAs suggest that the restriction enzyme digestion patterns of the regions of the rescued Rqm166 genomic DNA samples other than the m166 region appeared to be identical to those of the parental Rvm166 (data not shown). This observation suggests that the regions of the Rqm166 genome other than the $\mathrm{m} 166$ region remained intact and were identical to those of Rvm166. Thus, Rqm166 may represent a rescued virus for Rvm166.

Northern analyses of the m166 transcript expressed from Rvm166 and rescued virus Rqm166. Transcription from the target m166 region is expected to be disrupted due to the presence of the two transcription termination signals within the transposon (Fig. 1A). In particular, the region of the m166 open reading frame downstream from the transposon insertion site is not expected to be expressed. To determine whether this is the case, cytoplasmic RNAs were isolated from cells infected with the mutant virus at different time points (e.g., 4, 12, and $24 \mathrm{~h}$ ) postinfection. Northern analysis was carried out to examine the expression of the transcripts from the m166 open reading frame downstream from the transposon insertion site (Fig. 2). The probe (the $3^{\prime}$ probe) used in the Northern analyses contained the DNA sequence complementary to the $3^{\prime}$ $\mathrm{m} 166$ coding region that is within 200 nucleotides downstream from the site of the transposon insertion. A RNA species of about 2,000 nucleotides was detected in the RNA fractions 
(A)

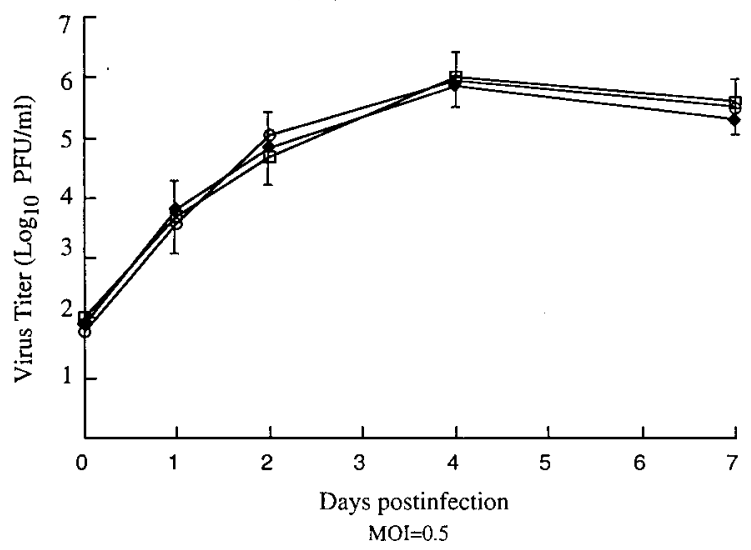

(B)

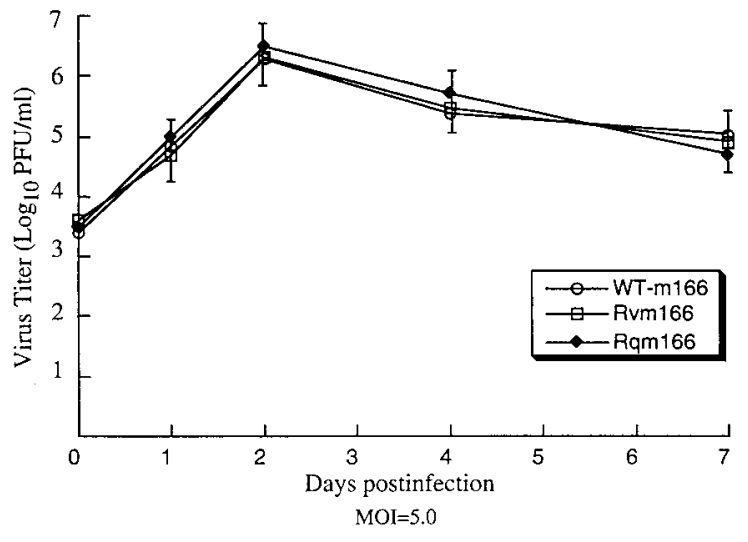

FIG. 3. Growth of MCMV mutants in tissue culture. Mouse NIH 3 T3 cells were infected with each virus at a MOI of either 0.5 PFU (A) or 5 PFU (B) per cell. At 0, 1, 2, 4, and 7 days postinfection, cells and culture media were harvested and sonicated. The viral titers were determined by plaque assays on NIH 3T3 cells. The values of the viral titer represent the average obtained from triplicate experiments. The standard deviation is indicated by the error bars.

isolated from cells that were infected with the wild-type Smith strain (Fig. 2, lane 2). This RNA species was also readily detected from cells infected with the Smith strain by using a DNA probe (the $5^{\prime}$ probe) complementary to the $5^{\prime}$-terminal sequence of m166 open reading frame that is within $300 \mathrm{nu}$ cleotides downstream from the m166 translational initiation site (data not shown). These results suggest that this $\sim 2-\mathrm{kb}$ RNA species represents the transcript expressed from the m166 open reading frame. However, this transcript was not detected in the RNA fractions isolated from cells infected with Rvm166 when the $3^{\prime}$ probe, which is complementary to the m166 coding region downstream from the site of the transposon insertion, was used in the Northern analyses (Fig. 2, lane 4). These observations suggest that the transcription from the m166 region downstream from the transposon insertion site was disrupted in Rvm166. Meanwhile, expression of the transcript was found in the RNA fractions from cells infected with the rescued virus Rqm166 (Fig. 2, lane 3). The level of MCMV M25 transcript $(8,49)$ was used as the internal control for the expression of the m166 transcript. As shown in Fig. 2, the levels of the M25 transcript detected in cells that were infected with Rvm166 and Rqm166 were found to be similar to that of M25 transcript in cells infected with the Smith strain (Fig. 2, lanes 5 to 8). Thus, the transposon insertion in Rvm166 appeared to disrupt the transcript expressed from the m166 open reading frame, whereas the expression of the transcript was restored in Rqm166.

Replication of Rvm166 and Rqm166 in vitro in tissue culture. The growth rates of the recombinant viruses in mouse NIH 3T3 cells were studied in order to determine whether these viruses had any growth defects in vitro. Cells were infected with these viruses at both low and high MOIs, and their growth rates were assayed by triplicate experiments. No significant difference was found in growth rates among Rvm166, Rqm166, and the Smith strain. For example, the peak titers of Rvm166 and Rqm166 were similar to those of the Smith strain under both high- and low-MOI infections (Fig. 3). Previous studies have shown that viral mutants with large deletions of 9- to $15-\mathrm{kb}$ DNA sequences that may encompass the m166 region replicated in NIH 3 T3 cells as well as the Smith stain $(1,43)$. Our results are consistent with these previous observations that m166 is probably not essential for viral growth in vitro.

Growth of recombinant viruses Rvm166 and Rqm166 in immunocompetent animals. To determine whether the disruption of m166 significantly affects viral replication in vivo, BALB/c-ByJ mice were injected intraperitoneally with $10^{4}$ PFU of Rvm166, Rqm166, or the wild-type Smith strain. At 1, $3,7,10,14$, and 21 days postinfection, salivary glands, lungs, spleens, livers, and kidneys were harvested, and the titers of the viral particles from these five organs were determined on NIH 3 T3 cells. These organs are among the major targets for MCMV infection $(16,18,26)$. At days 14 and 21 days postinfection, the titers of Rvm 166 found in the salivary glands were about 500- and 5,000-fold lower than those of the Smith strain, respectively (Fig. 4A). Moreover, the peak titers of Rvm166 found in the lungs, spleens, livers, and kidneys of the infected animals (at 10 days postinfection) were at least 100-, 50-, 80-, and 30-fold lower than the titers in the same organs from the animals infected with the Smith strain (Fig. 4B to E). In contrast, the titers of the rescued virus Rqm166 found in the same organs were similar to the titers of the Smith strain. Previous studies have shown that the presence of the transposon sequence per se within the viral genome does not significantly affect viral growth in BALB/c mice in vivo (49). Thus, these results suggest that the growth deficiency of Rvm166 in the organs examined is due to the disruption of m166 and that open reading frame m166 is important for optimal viral growth in vivo, at least in these organs in BALB/c mice.

Virulence and growth of viral mutants in immunodeficient SCID mice. Immunodeficient animals, including the CB17 SCID mice, are extremely susceptible to MCMV infection (15, 29, 31, 33). Analysis of viral replication in these mice therefore serves as an excellent model for determining the virulence of different MCMV strains and mutants and for studying the mechanism of how they cause opportunistic infections in immunocompromised hosts. To determine whether the m166 
(A) Salivary gland

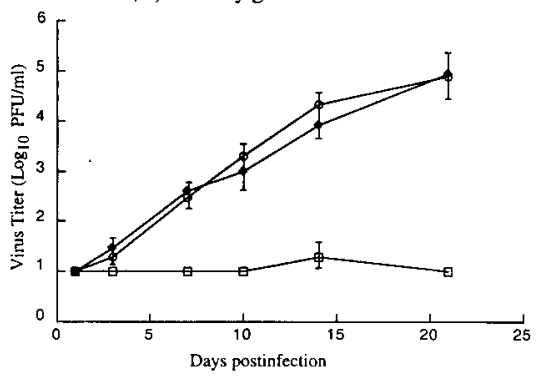

(B) Lung

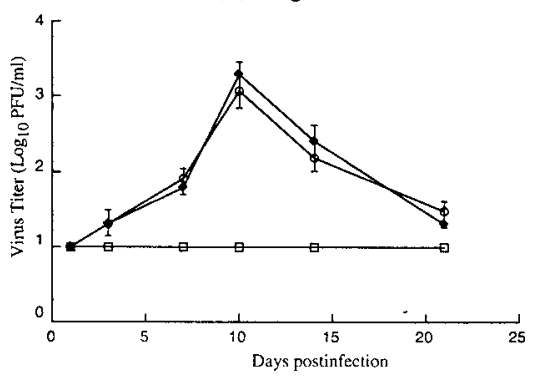

(C) Spleen

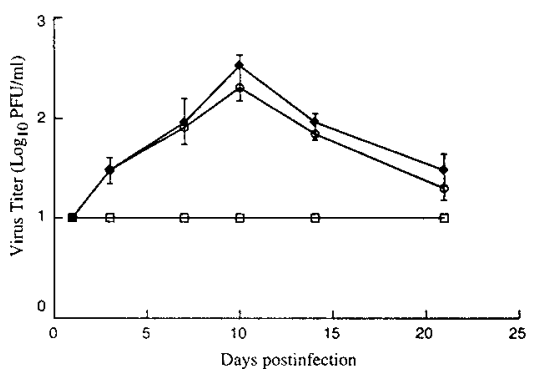

(D) Liver

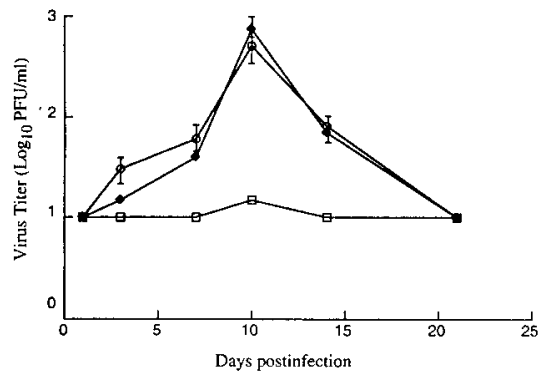

(E) Kidney

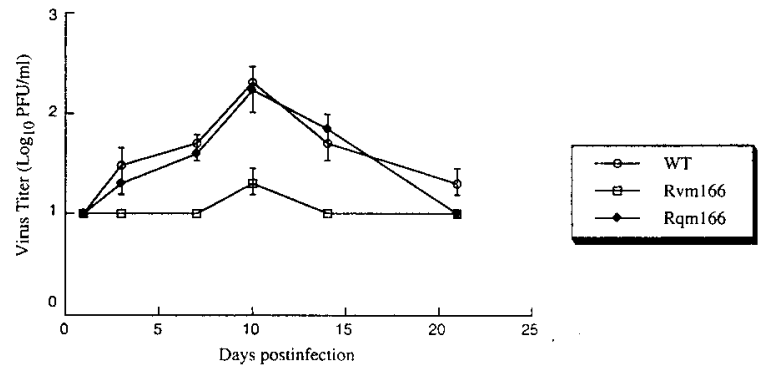

FIG. 4. Titers of MCMV mutants in the salivary glands (A), lungs (B), spleens (C), livers (D), and kidneys (E) of the infected BALB/c mice. $\mathrm{BALB} / \mathrm{c}-\mathrm{ByJ}$ mice were infected intraperitoneally with $10^{4} \mathrm{PFU}$ of each virus. At 1, 3, 7, 10, 14, and 21 days postinfection, the animals (three mice per group) were sacrificed, and the organs were collected and sonicated. The viral titers in the tissue homogenates were determined by standard plaque assays in NIH 3T3 cells. The viral titers represent the average obtained from triplicate experiments. The error bars indicate the standard deviation. Error bars that are not evident indicate that the standard deviation was less than or equal to the height of the symbols. The limit of detection was $10 \mathrm{PFU} / \mathrm{ml}$ of the tissue homogenate.

open reading frame plays a significant role in CMV virulence, we compared the survival rates of the animals infected with Rvm166 and those of Rqm166 and the wild-type Smith strain. For each virus, five SCID mice were injected intraperitoneally with $10^{4}$ PFU of Rvm166, Rqm166, or the Smith strain. The viral inoculum used for the infection of animals were prepared by growing the viruses in NIH 3T3 cells. All of the mice that were infected with either Smith strain or Rqm166 died within 26 to 27 days postinfection (Fig. 5). In contrast, no animals infected with Rvm166 died even after 50 days postinfection (Fig. 5). Indeed, all animals infected with Rvm166 remained alive even after 90 days postinfection. This observation indicated that Rvm166 was severely attenuated in viral virulence in killing SCID mice. It has been demonstrated in our laboratory that the presence of the transposon sequence per se within the viral genome does not significantly affect MCMV virulence in killing SCID mice (49). Thus, these results suggest that disruption of the m166 open reading frame results in a complete loss of viral virulence and that m166 is essential for MCMV virulence in SCID mice.

To further study the pathogenesis of the mutant virus in these immunodeficient animals, the replication of Rvm166 in different organs of the animals was studied during a 21-day infection period before the mortality of the infected animals became apparent. In these experiments, SCID mice were injected intraperitoneally with $10^{4} \mathrm{PFU}$ of each virus (Rvm166, Rqm166, or the wild-type Smith strain). At 1, 3, 7, 10, 14, and 21 days postinfection, three mice from each virus group were sacrificed, and the salivary glands, lungs, spleens, livers, and kidneys were harvested. The levels of viral growth in these five organs were determined by assaying the viral titers in the organs. The titers of Rqm166 in each of the organs examined were similar to those of the Smith strain (Fig. 6). In contrast, the titers of the mutant virus Rvm166 were consistently lower than those of the wild-type virus at every time point examined. At 21 days postinfection, the titers of Rvm166 in the salivary glands, lungs, spleens, livers, and kidneys of the infected animals were lower than the titers of the wild-type virus by about

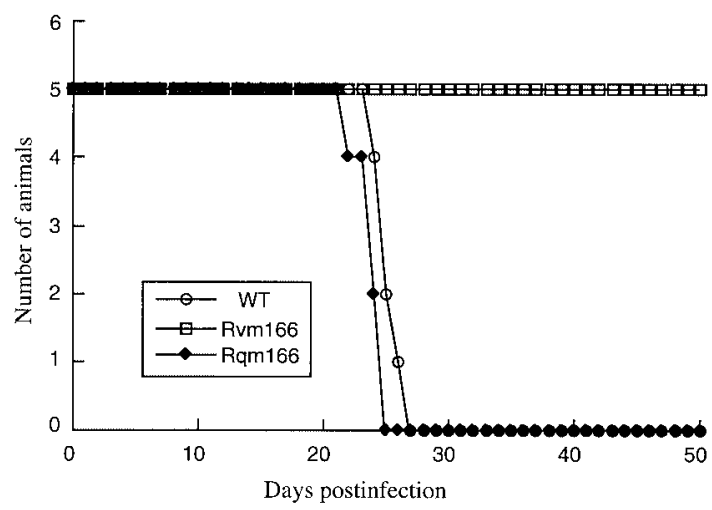

FIG. 5. Survival rate of the SCID mice infected with the Smith strain, Rvm166, and Rqm166. CB17 SCID mice (five animals per group) were infected intraperitoneally with $10^{4} \mathrm{PFU}$ of each virus. The mortality of the mice was monitored for at least 50 days postinfection, and survival rates were determined. 
(A) Salivary gland

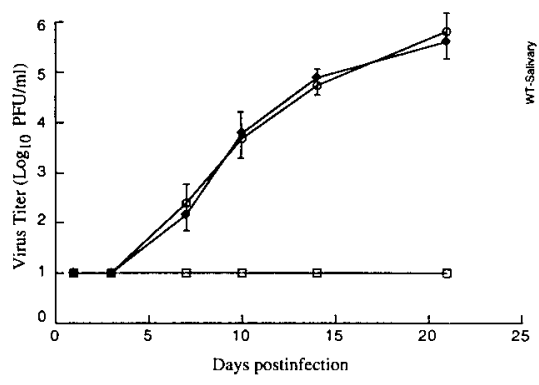

(B) Lung

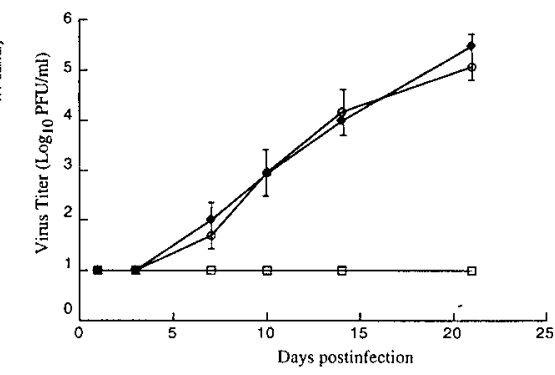

(C) Spleen

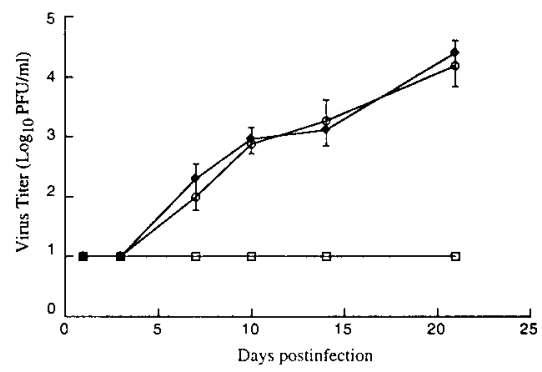

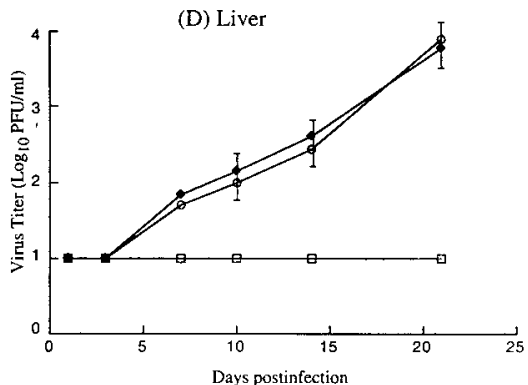

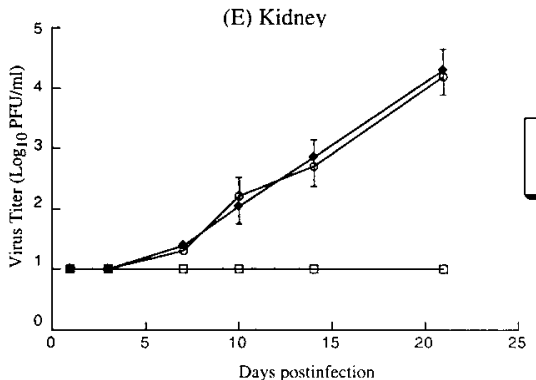

(E) Kidney

Days postinfection

FIG. 6. Titers of MCMV mutants in the salivary glands (A), lungs (B), spleens (C), livers (D), and kidneys (E) of the infected SCID mice. CB17 SCID mice were infected intraperitoneally with $10^{4} \mathrm{PFU}$ of each virus. At $1,3,7,10,14$, and 21 days postinfection, the animals (three mice per group) were sacrificed, and the organs were collected and sonicated. The viral titers in the tissue homogenates were determined by standard plaque assays in NIH $3 \mathrm{~T} 3$ cells. The viral titers represent the average obtained from triplicate experiments. The error bars indicate the standard deviation. Error bars that are not evident indicate that the standard deviation was less than or equal to the height of the symbols. The limit of detection was $10 \mathrm{PFU} / \mathrm{ml}$ of the tissue homogenate.

30,000-, 10,000-, 1,000-, 300-, and 800-fold, respectively (Fig. 6). Therefore, Rvm166 appears to be significantly deficient in growth in the organs of the immunodeficient animals. Previous studies have shown that the presence of the transposon sequence per se within the viral genome does not significantly affect viral growth in SCID mice in vivo (49). Thus, these results suggest that the attenuated growth of Rvm166 in these organs is probably due to the disruption of m166 and that open reading frame m166 is required for optimal growth of MCMV in these organs in immunodeficient hosts.

The genome and transposon mutation of Rvm166 is stable during viral growth in SCID mice. It has been reported that MCMV mutants with an insertional sequence are not stable and generate spontaneous mutations during replication in vitro and in vivo (23; Abenes et al., unpublished). Indeed, a viral mutant with a spontaneous deletion at the viral 3 '-terminal region encompassing $\mathrm{m} 166$ has been reported after extensive passage of the virus in tissue-cultured cells (1). It is possible that the transposon sequence in Rvm166 is not stable during viral replication in vivo and the introduction of an adventitious mutation may be responsible for the observed phenotypes of the virus in animals. To address this issue, the salivary glands and the lungs of the Rvm166-infected SCID mice were harvested at 21 days postinfection. The viruses were subsequently recovered from these organs by infecting NIH 3T3 cells with the sonicated tissue homogenates. Viral DNAs were purified from the infected cells, and their restriction digestion patterns were analyzed in agarose gels. Moreover, we also performed Southern analysis of the Rvm166 viral DNAs with a DNA probe that contained the transposon and the m166 open reading frame sequence. The results showed that no change in the hybridization patterns of Rvm166 occurred as a result of viral growth in animals for 21 days (Fig. 7). Moreover, the overall EcoRI digestion patterns of Rvm166 DNA isolated from either infected cultured cells or animals were identical to those of the original recombinant virus Rvm166, as visualized by ethidium bromide staining of the viral DNAs (data not shown). Thus, the transposon insertion in Rvm166 appeared to be stable, and the genome of Rvm166 remained intact during replication in the infected animals.

\section{DISCUSSION}

In this study, we characterized a recombinant MCMV virus that contained the transposon insertional mutation at open reading frame m166 both in cell culture and in animals. Since HCMV does not contain a sequence homologue of m166, the function of m166 may be involved in species-specific interactions between MCMV and its natural host, mice. These interactions may not be found between HCMV and its respective host, humans. Our study indicates that a disruption of the $\mathrm{m} 166$ open reading frame results in severely reduced growth of the virus in both BALB/c and SCID mice and abolishes viral virulence in killing SCID mice. Thus, our results provide the first direct evidence to suggest that m166 probably encodes a virulence factor and is required for MCMV virulence in killing SCID mice and for optimal growth in vivo.

Although it is possible that the functional m166 protein 
product might be synthesized from the transposon-disrupted region, several lines of evidence strongly suggest that this is not the case. First, the transposon sequence was inserted into the coding sequence of the m166 open reading frame (Fig. 1B). Second, transcription from the region downstream from the transposon insertion site was not detected in cells infected with the mutant virus (Fig. 2). Thus, the region of the target open reading frame downstream from the transposon insertion site, which includes $>65 \%$ of the m166 coding sequence, was not expressed. Therefore, it is likely that no functional m166 protein was expressed from the viral mutant.

The function of m166 is currently unknown. Indeed, to our knowledge, neither the transcript nor the protein product coded by this open reading frame has been reported. Our results indicate that a transcript of about 2,000 nucleotides is expressed from the m166 open reading frame. The growth rate of Rvm166 in NIH 3T3 cells was not significantly different from that of the Smith strain (Fig. 3). These results provide the evidence that the carboxyl 252-amino-acid sequence of m166 is not essential for viral replication in vitro. Moreover, these results are consistent with previous observations $(1,43)$ that $\mathrm{m} 166$ is probably dispensable for MCMV growth in vitro in NIH 3 T3 cells.

Our results indicate that Rvm166 was significantly attenuated in replication in the salivary glands, lungs, spleens, livers, and kidneys of both the BALB/c and SCID mice that were intraperitoneally infected. For example, at 21 days postinfection, the titers of Rvm166 in the salivary glands, lungs, spleens, liver, and kidneys of the SCID mice were $<2 \times 10^{1} \mathrm{PFU} / \mathrm{ml}$ of organ homogenates. In contrast, the titers of the Smith strain, as well as rescued virus Rqm166 in the salivary glands, lungs, spleens, livers, and kidneys of the infected animals were about $5 \times 10^{5}, 1 \times 10^{5}, 1 \times 10^{4}, 3 \times 10^{3}$, and $8 \times 10^{3} \mathrm{PFU} / \mathrm{ml}$ of organ homogenates, respectively (Fig. 6). Moreover, no death was found in SCID mice infected with Rvm166 up to 90 days postinfection, whereas all of the mice infected with the Smith strain or Rqm166 were found dead within 27 days postinfection (Fig. 5). These results strongly suggest that $\mathrm{m} 166$ is a viral determinant for MCMV growth in vivo in these animals and for viral virulence in killing SCID mice.

It is possible that the observed change in the levels of replication of the mutant is due to other adventitious mutations accumulated during the construction and growth of the recombinant virus in cultured cells or in animals. However, several lines of evidence strongly suggest that this is unlikely. First, rescued virus Rqm166 replicated equally well in the salivary glands, lungs, spleens, livers, and kidneys of the infected $\mathrm{BALB} / \mathrm{c}$ and SCID mice as the wild-type virus (Fig. 4 and 6). Moreover, the rescued virus exhibited a similar level of virulence as the wild-type virus (Fig. 5). The genomic sequence of m166 and its expression in the rescued virus were restored (Fig. 1 and 2). These observations suggest that the transposon insertion, rather than a second mutation, is responsible for the observed attenuation of Rvm166 replication and virulence in the infected animals. Second, our previous studies indicated that a virus mutant (i.e., Rvm09) with transposon insertion at the m09 open reading frame replicated equally well in both $\mathrm{BALB} / \mathrm{c}$ and SCID mice as the wild-type virus (49). Moreover, mutant Rvm09 exhibited a similar level of virulence in killing SCID mice as the wild-type virus. These observations indicated

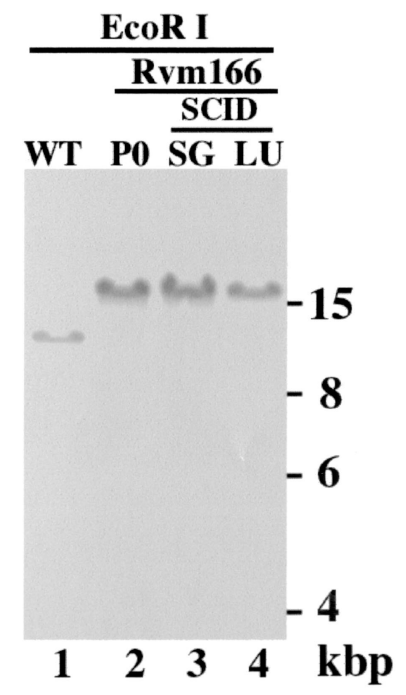

FIG. 7. Stability of the transposon mutation of Rvm166 in SCID mice. Viral DNAs were either isolated from cells that were infected with Rvm166 (MOI <0.01) and allowed to grow in culture for 5 days (P0) (lane 2) or from cells that were infected with the virus collected from the salivary glands (SG, lanes 3 ) or lungs (LU, lane 4 ) of the SCID mice 21 days after intraperitoneal inoculation with $10^{4}$ PFU of Rvm166. Southern analyses of the viral DNA fractions digested with EcoRI are shown. The DNA of the wild-type virus (WT) is shown in lane 1 . The ${ }^{32} \mathrm{P}$-radiolabeled probe was derived from the plasmid containing the transposon and the m166 open reading frame sequence.

that the transposon sequence per se in the viral genome does not significantly affect viral replication and virulence in these animals (49). Third, the transposon insertion was stable during replication in animals. There was no change in the hybridization patterns of the DNAs from the mutant virus recovered from the salivary glands and lungs of the infected animals at 21 days postinfection (Fig. 7). Moreover, the EcoRI digestion patterns of the Rvm166 mutant DNAs, other than the region with the inserted transposon, appeared to be identical to those of the wild-type virus DNA (data not shown). Thus, the observed change of the level of Rvm166 replication and virulence in the infected animals is probably due to the disruption of the m166 expression as a result of the transposon insertion.

Our results are consistent with previous observations with viral mutants that contained large deletions at the 3 '-terminal region that may encompass the $\mathrm{m} 166$ open reading frame. These mutants, which contained a deletion of a 9.6- and $15.8-\mathrm{kb}$ sequence at the 3 '-terminal region, respectively, exhibited attenuated growth in spleens and salivary glands of the infected BALB/c mice $(1,43)$. However, whether these mutants are deficient in growth in immunodeficient mice has not been reported. Equally unclear is whether their virulence in killing animals is attenuated. It is possible that 70 to $100 \%$ of the m166 sequence might remain in these viral mutants $(1,43)$. Meanwhile, the large sizes of the deletions in these mutants resulted in the removal of multiple open reading frames. It is unclear whether the observed attenuated phenotypes are either due to the mutation of multiple genes or a single specific open reading frame $(1,43)$. Our results provide the direct evidence that a single mutation at the m166 open reading frame results in a significant reduction of viral growth and 
virulence in vivo. Moreover, our study indicates for the first time that $\mathrm{m} 166$ is a virulence factor required for viral killing in SCID mice.

A key question from our results is how the lack of m166 leads to a change in the level of virulence and growth. It is possible that the defect of $\mathrm{m} 166$ inhibits the spread of the virus to the target organs, entry into permissive cells, or full replication inside infected cells. Given the fact that Rvm166 replicates normally in NIH 3T3 cells, it is conceivable that a viral mutant with disruption of m166 exhibits a defect in certain steps of viral replication in vivo in specific cells other than fibroblasts of particular organs and tissues. This defect may lead to slow growth of the viral mutant in the organs and, consequently, may contribute to severe reduction of growth of the viral mutant in vivo. Alternatively, m166 may be involved in virus-host interactions and play an important role in modulating the host cells for optimal viral replication. Although little is currently known about the molecular mechanism of m166 in supporting viral growth and virulence in vivo, its sequence predicts that $\mathrm{m} 166$ encodes a putative membrane glycoprotein (32). Attempts have been made to compare the in vivo phenotypes of Rvm166 with the phenotypes of other viral mutants, including those that were generated in our laboratory by transposon insertion at different loci of the viral genome. It appears that the levels of attenuation in the growth and virulence of Rvm166 in the infected SCID mice are very similar to those of the viral mutant that contains a transposon mutation in the M37 open reading frame, which is also believed to encode a membrane glycoprotein (19). Although the molecular mechanism of M37 in supporting viral growth and virulence is currently unknown, its HCMV homolog, UL37, is believed to encode an antiapoptotic factor localized in mitrochondrial membrane and is also able to transactivate both viral and cellular promoters $(7,13)$. While $\mathrm{m} 166$ does not share extensive sequence homology with M37, it will be interesting to determine whether these putative membrane glycoproteins function in a similar way in supporting optimal viral growth and virulence in vivo. Further studies on in vitro and in vivo replication of Rvm166 and other viral mutants (e.g., the mutant with a transposon insertion at M37) will further provide insight into the functions of viral genes in vivo in MCMV virulence and pathogenesis.

\section{ACKNOWLEDGMENTS}

We thank John Kim, Karen Chan, Dinesh Dhanaraj, and Ada Tam for excellent technical assistance.

F.L. is a Pew Scholar in Biomedical Sciences, a scholar of the Leukemia and Lymphoma Society, and a recipient of an American Heart Association Established Investigator Award. This research was, in part, supported by the March of Dimes National Birth Defects Foundation and the NIH.

\section{REFERENCES}

1. Boname, J. M., and J. K. Chantler. 1992. Characterization of a strain of murine cytomegalovirus which fails to grow in the salivary glands of mice. J. Gen. Virol. 73:2021-2029.

2. Borst, E., G. Hahn, U. H. Koszinowski, and M. Messerle. 1999. Cloning of the human cytomegalovirus (HCMV) genome as an infectious bacterial chromosome in Escherichia coli: a new approach for construction of HCMV mutants. J. Virol. 73:8320-8329.

3. Bruggeman, C. A., H. J. Marjorie, and G. Nelissen-Vrancken. 1999. Cytomegalovirus and atherogenesis. Antiviral Res. 43:135-144.

4. Brune, W., C. Menard, U. Hobom, S. Odenbreit, M. Messerle, and U. H. Koszinowski. 1999. Rapid identification of essential and nonessential her- pesvirus genes by direct transposon mutagenesis. Nat. Biotechnol. 17:360364

5. Burns, N., B. Grimwade, P. B. Ross-Macdonald, E. Y. Choi, K. Finberg, G. S. Roeder, and M. Snyder. 1994. Large-scale analysis of gene expression, protein localization, and gene disruption in Saccharomyces cerevisiae. Genes Dev. 8:1087-1105.

6. Chee, M. S., A. T. Bankier, S. Beck, R. Bohni, C. M. Brown, R. Cerny, T. Horsnell, C. A. Hutchison, T. Kouzarides, and J. A. Martignetti. 1990. Analysis of the protein-coding content of the sequence of human cytomegalovirus strain AD169. Curr. Top. Microbiol. Immunol. 154:125-169.

7. Colberg-Poley, A. M., L. D. Santomenna, P. P. Harlow, P. A. Benfield, and D. J. Tenney. 1992. Human cytomegalovirus US3 and UL36-38 immediateearly proteins regulate gene expression. J. Virol. 66:95-105.

8. Dallas, P. B., P. A. Lyons, J. B. Hudson, A. A. Scalzo, and G. R. Shellam. 1994. Identification and characterization of a murine cytomegalovirus gene with homology to the UL25 open reading frame of human cytomegalovirus. Virology 200:643-650.

9. Danesh, J., R. Collins, and R. Peto. 1997. Chronic infections and coronary heart disease: is there a link? Lancet 350:430-436.

10. Delecluse, H. J., T. Hilsendegen, D. Pich, R. Zeidler, and W. Hammerschmidt. 1998. Propagation and recovery of intact, infectious Epstein-Barr virus from prokaryotic to human cells. Proc. Natl. Acad. Sci. USA 95:82458250 .

11. Fleming, P., N. Davis-Poynter, M. Degli-Esposti, E. Densley, J. Papadimitriou, G. Shellam, and H. Farrell. 1999. The murine cytomegalovirus chemokine homolog, m131/129, is a determinant of viral pathogenicity. J. Virol. 73:6800-6809.

12. Fowler, K. B., S. Stagno, R. F. Pass, W. J. Britt, T. J. Boll, and C. A. Alford. 1992. The outcome of congenital cytomegalovirus infection in relation to maternal antibody status. N. Engl. J. Med. 326:663-667.

13. Goldmacher, V. S., L. M. Bartle, A. Skaletskaya, C. A. Dionne, N. L. Kedersha, C. A. Vater, J. Han, R. J. Lutz, S. Watanabe, E. D. McFarland, E. D. Kieff, E. S. Mocarski, and T. Chittenden. 1999. A cytomegalovirus-encoded mitochondria-localized inhibitor of apoptosis structurally unrelated to Bcl-2. Proc. Natl. Acad. Sci. USA 96:12536-12541.

14. Greaves, R. F., J. M. Brown, J. Vieira, and E. S. Mocarski. 1995. Selectable insertion and deletion mutagenesis of the human cytomegalovirus genome using the Escherichia coli guanosine phosphoribosyl transferase (gpt) gene. J. Gen. Virol. 76:2151-2160.

15. Grundy, J. E., and C. J. Melief. 1982. Effect of $\mathrm{Nu} / \mathrm{Nu}$ gene on genetically determined resistance to murine cytomegalovirus. J. Gen. Virol. 61:133-136.

16. Hudson, J. B. 1979. The murine cytomegalovirus as a model for the study of viral pathogenesis and persistent infections. Arch. Virol. 62:1-29.

17. Jenkins, F. J., M. J. Casadaban, and B. Roizman. 1985. Application of the mini-Mu-phage for target-sequence-specific insertional mutagenesis of the herpes simplex virus genome. Proc. Natl. Acad. Sci. USA 82:4773-4777.

18. Jordan, M. C. 1983. Latent infection and the elusive cytomegalovirus. Rev. Infect. Dis. 5:205-215.

19. Lee, M., J. Xiao, E. Haghjoo, T. Tuong, G. Abenes, X. Zhan, W. Dunn, and F. Liu. 2000. Murine cytomegalovirus containing a mutation at open reading frame M37 is severely attenuated in growth and virulence in vivo. J. Virol. 74:11099-11107.

20. Liu, F., and B. Roizman. 1991. The herpes simplex virus 1 gene encoding a protease also contains within its coding domain the gene encoding the more abundant substrate. J. Virol. 65:5149-5156.

21. Liu, F. Y., and B. Roizman. 1991. The promoter, transcriptional unit, and coding sequence of herpes simplex virus 1 family 35 proteins are contained within and in frame with the UL26 open reading frame. J. Virol. 65:206-212.

22. MacDonald, M. R., M. W. Burney, S. B. Resnick, and H. I. Virgin. 1999. Spliced mRNA encoding the murine cytomegalovirus chemokine homolog predicts a beta chemokine of novel structure. J. Virol. 73:3682-3691.

23. Manning, W. C., C. A. Stoddart, L. A. Lagenaur, G. B. Abenes, and E. S. Mocarski. 1992. Cytomegalovirus determinant of replication in salivary glands. J. Virol. 66:3794-3802.

24. Melnick, J. L., E. Adam, and M. E. DeBakey. 1995. Cytomegalovirus and atherosclerosis. Bioessays 17:899-903.

25. Messerle, M., I. Crnkovic, W. Hammerschmidt, H. Ziegler, and U. H. Koszinowski. 1997. Cloning and mutagenesis of a herpesvirus genome as an infectious bacterial artificial chromosome. Proc. Natl. Acad. Sci. USA 94: $14759-14763$.

26. Mocarski, E. S., and C. T. Courcelle. 2001. Cytomegaloviruses and their replication, p. 2629-2673. In D. M. Knipe and P. M. Howley (ed.), Fields virology. Lippincott/The Williams and Wilkins Co., Philadelphia, Pa.

27. Mocarski, E. S., L. E. Post, and B. Roizman. 1980. Molecular engineering of the herpes simplex virus genome: insertion of a second L-S junction into the genome causes additional genome inversions. Cell 22:243-255.

28. Mulligan, R. C., and P. Berg. 1981. Selection for animal cells that express the Escherichia coli gene coding for xanthine-guanine phosphoribosyltransferase. Proc. Natl. Acad. Sci. USA 78:2072-2076.

29. Okada, M., and Y. Minamishima. 1987. The efficacy of biological response modifiers against murine cytomegalovirus infection in normal and immunodeficient mice. Microbiol. Immunol. 31:45-57. 
30. Pass, R. F. 2001. Cytomegalovirus, p. 2675-2706. In D. M. Knipe and P. M Howley (ed.), Fields virology. Lippincott/The Williams and Wilkins Co. Philadelphia, $\mathrm{Pa}$

31. Pollock, J. L., and H. W. Virgin. 1995. Latency, without persistence, of murine cytomegalovirus in the spleen and kidney. J. Virol. 69:1762-1768.

32. Rawlinson, W. D., H. E. Farrell, and B. G. Barrell. 1996. Analysis of the complete DNA sequence of murine cytomegalovirus. J. Virol. 70:8833-8849.

33. Reynolds, R. P., R. J. Rahija, D. I. Schenkman, and C. B. Richter. 1993 Experimental murine cytomegalovirus infection in severe combined immunodeficient mice. Lab. Anim. Sci. 43:291-295.

34. Ross-Macdonald, P., P. S. Coelho, T. Roemer, S. Agarwal, A. Kumar, R. Jansen, K. H. Cheung, A. Sheehan, D. Symoniatis, L. Umansky, M. Heidtman, F. K. Nelson, H. Iwasaki, K. Hager, M. Gerstein, P. Miller, G. S. Roeder, and M. Snyder. 1999. Large-scale analysis of the yeast genome by transposon tagging and gene disruption. Nature 402:413-418.

35. Saederup, N., Y. C. Lin, D. J. Dairaghi, T. J. Schall, and E. S. Mocarski. 1999. Cytomegalovirus-encoded beta chemokine promotes monocyte-associated viremia in the host. Proc. Natl. Acad. Sci. USA 96:10881-10886.

36. Saeki, Y., T. Ichikawa, A. Saeki, E. A. Chiocca, K. Tobler, M. Ackermann, X. O. Breakefield, and C. Fraefel. 1998. Herpes simplex virus type 1 DNA amplified as bacterial artificial chromosome in Escherichia coli: rescue of replication-competent virus progeny and packaging of amplicon vectors. Hum. Gene Ther. 9:2787-2794.

37. Seifert, H. S., E. Y. Chen, M. So, and F. Heffron. 1986. Shuttle mutagenesis a method of transposon mutagenesis for Saccharomyces cerevisiae. Proc. Natl. Acad. Sci. USA 83:735-739.

38. Smith, G. A., and L. W. Enquist. 1999. Construction and transposon mutagenesis in Escherichia coli of a full-length infectious clone of pseudorabies virus, an alphaherpesvirus. J. Virol. 73:6405-6414.

39. Smith, G. A., and L. W. Enquist. 2000. A self-recombining bacterial artificial chromosome and its application for analysis of herpesvirus pathogenesis. Proc. Natl. Acad. Sci. USA 97:4873-4878.

40. Spaete, R. R., and E. S. Mocarski. 1987. Insertion and deletion mutagenesis of the human cytomegalovirus genome. Proc. Natl. Acad. Sci. USA 84:72137217 .
41. Stavropoulos, T. A., and C. A. Strathdee. 1998. An enhanced packaging system for helper-dependent herpes simplex virus vectors. J. Virol. 72:71377143.

42. Streblow, D. N., S. L. Orloff, and J. A. Nelson. 2001. Do pathogens accelerate atherosclerosis? J. Nutr. 131:2798S-2804S.

43. Thale, R., U. Szepan, H. Hengel, G. Geginat, P. Lucin, and U. H. Koszinowski. 1995. Identification of the mouse cytomegalovirus genomic region affecting major histocompatibility complex class I molecule transport. J. Virol. 69:6098-6105.

44. Vieira, J., H. E. Farrell, W. D. Rawlinson, and E. S. Mocarski. 1994. Genes in the HindIII $\mathbf{J}$ fragment of the murine cytomegalovirus genome are dispensable for growth in cultured cells: insertion mutagenesis with a lacZ/gpt cassette. J. Virol. 68:4837-4846.

45. Wagner, M., S. Jonjic, U. H. Koszinowski, and M. Messerle. 1999. Systematic excison of vector sequences from the BAC-cloned herpesvirus genome during virus reconstitution. J. Virol. 73:7056-7060.

46. Weber, P. C., M. Levine, and J. C. Glorioso. 1987. Rapid identification of nonessential genes of herpes simplex virus type 1 by Tn 5 mutagenesis. Science 236:576-579.

47. Wiertz, E. J., T. R. Jones, L. Sun, M. Bogyo, H. J. Geuze, and H. L. Ploegh. 1996. The human cytomegalovirus US11 gene product dislocates MHC class I heavy chains from the endoplasmic reticulum to the cytosol. Cell 84:769779 .

48. Zhan, X., G. Abenes, M. Lee, I. VonReis, C. Kittinunvorakoon, P. RossMacdonald, M. Snyder, and F. Liu. 2000. Mutagenesis of murine cytomegalovirus using a Tn3-based transposon. Virology 266:264-274.

49. Zhan, X., M. Lee, J. Xiao, and F. Liu. 2000. Construction and characterization of murine cytomegaloviruses that contain a transposon insertion at open reading frames m09 and M83. J. Virol. 74:7411-7421.

50. Zhou, Y. F., E. Guetta, Z. X. Yu, T. Finkel, and S. E. Epstein. 1996. Human cytomegalovirus increases modified low-density lipoprotein uptake and scavenger receptor mRNA expression in vascular smooth muscle cells. J. Clin. Investig. 98:2129-2138. 